\title{
CONTROLE TUMORAL POR MEIO DE INFUSÃO DE AGENTES QUIMIOTERÁPICOS
}

\author{
Fernando da Silva Borges*, Kelly Cristiane Iarosz ${ }^{\dagger}$, Antonio Marcos Batista ${ }^{\ddagger}$, Sergio \\ Roberto LOpes ${ }^{\S}$, RicARdo Luiz Viana ${ }^{\S}$ \\ *Pós-Graduação em Ciências \\ Universidade Estadual de Ponta Grossa \\ Ponta Grossa, Paraná, Brasil \\ $\dagger$ Institute for Complex Systems and Mathematical Biology \\ University of Aberdeen \\ Aberdeen, Scotland,United Kingdom \\ ${ }^{\ddagger}$ Departamento de Matemática e Estatística \\ Universidade Estadual de Ponta Grossa \\ Ponta Grossa, Paraná, Brasil \\ $\S$ Departamento de Física \\ Universidade Federal do Paraná \\ Curitiba, Paraná, Brasil
}

\author{
Emails: fernandodasilvaborges@gmail.com, kiarosz@gmail.com, \\ antoniomarcosbatista@gmail.com, lopes@fisica.ufpr.br, viana@fisica.ufpr.br
}

\begin{abstract}
One possible way to contain the growth of a tumor is the chemical infusion that affect cell function. This process is known as chemotherapy, and it consists of a drug treatment through some protocol. In this work we propose a mathematical model with interactions among the immune system, tumor and chemotherapy. We extend the model of Banerjee and Sarkar by adding the chemotherapy. A continuous and pulsed chemotherapy are analyzed. Our main results show there is cancer suppression when applying continuous chemotherapy without vanishing the lymphocites, and that pulsed chemotherapy protocols maximize elimination of cancerous cells while minimise the negative effects on normal cells.
\end{abstract}

Keywords- Cancer, differential equations, chemotherapy.

Resumo- Uma das possíveis formas de conter o crescimento de um tumor é a infusão de substâncias químicas que afetam o funcionamento celular. Esse processo é conhecido como quimioterapia, e consiste em um tratamento que utiliza de uma forma geral, combinações de drogas aplicadas por meio de protocolos médicos. Neste trabalho propõe-se modelar interações do sistema imunológico, tumores e efeitos da quimioterapia por meio de equações diferenciais. Para tanto, estudou-se o modelo proposto por Banerjee e Sarkar, adicionando-se ao mesmo termos que representam a quimioterapia. No desenvolvimento do trabalho, apresentam-se análises da aplicação de quimioterapia na forma contínua e na forma periódica. Com o modelo proposto observou-se a supressão do câncer aplicando-se quimioterapia continuamente, sem a eliminação de linfócitos e obteve-se protocolos de quimioterapia que maximizam a eliminação das células cncerígenas enquanto minimizam os efeitos negativos nas células normais.

Palavras-chave - Câncer, equações diferenciais, quimioterapia.

\section{Introdução}

Tumor é um aumento anormal de uma parte ou da totalidade de um tecido. Sua origem tem início em uma célula defeituosa que reproduz outras com a mesma deformidade, e assim por diante, fazendo com que um tumor cresça. Os tumores podem ser classificados como benignos ou malignos. Os tumores benignos se diferenciam dos malignos pela incapacida de metástase. A maioria dos tumores benignos crescem como massas coesivas em expansão, permanecendo situadas em seu local de origem.

Tumor maligno ou câncer é o nome dado a um conjunto de mais de 100 doenças que apresentam um ponto comum, o crescimento desordenado de células que invadem os tecidos e órgãos, estas células podem espalhar-se para outras regiões do corpo e dividir-se rapidamente formando tumores (Otto, 2002).

Uma das principais defesas do organismo contra vírus, bactérias intracelulares e tumores é a destruição de células infectadas ou tumorais por linfócitos T citotóxicos (LTC). Os LTC são capazes de aniquilar células, induzindo a morte celular programada, processo celular conhecido como apoptose (Otto, 2002). Para que o processo biológico de ativação ocorra com eficiência, os LTC precisam receber estímulos gerados por linfócitos $\mathrm{T}$ auxiliares (LTA), tais estímulos ocorrem por meio da liberação de citocinas. Esse processo não é instantâneo, há vários processos citológicos envolvidos que necessitam de um tempo para ocorrer (Wodarz et al., 1998; Iarosz et al., 2011).

Sarkar e Banerjee (Sarkar and Banerjee, 2005; Banerjee and Sarkar, 2008) desenvolveram um mo- 
delo para descrever a dinâmica de tumor considerando a interação das células do sistema imunológico (linfócitos T), que destroem as células do tumor maligno, como um relacionamento de predador-presa. Consideraram dois estados de linfócitos T: em caça (LTC) e em repouso (LTA). Estudaram o sistema sob flutuações externas, propuseram certos limites úteis para o controle o crescimento do tumor maligno, analisaram o efeito do tempo de atraso e compararam os resultados com situações reais.

Uma das formas mais comum e eficaz do tratamento do câncer é a utilização de compostos químicos, chamados quimioterápicos. Os agentes quimioterápicos, utilizados no tratamento do câncer, afetam tanto as células cancerígenas como as normais, podendo provocar vários efeitos colaterais, tais como perda de cabelo, náuseas, vômitos, diarréia, problemas instestinais e anemia. Outro efeito colateral comum, é o surgimento de infecções devido a diminuição do número de leucócitos, que são responsáveis pela defesa contra microrganismos (INCA, 2011).

Vários modelos presentes na literatura estudam a evolução do câncer incluindo a quimioterapia (Dorr and VonHoff, 1994; Liu and Freedman, 2005; Villasana and Radunskaya, 2003; Pillis et al., 2006; Engelhart et al., 2011). Um modelo simples e interessante, para taxa de variação da quantidade de quimioterapia, foi proposto por Pinho e colaboradores (2002) (Pinho et al., 2002). Nesse trabalho consideraram um sistema de equações que simulam as interações entre células normais, células cancerígenas e um agente quimioterápico.

O tratamento quimioterápico geralmente consiste em aplicações periódicas de agente quimioterápicos (AQ). Para cada caso específico de câncer designa-se um protocolo para a quimioterapia (INCA, 2011). A taxa de infusão do $\mathrm{AQ}(\Delta)$ está relacionada com a aplicação da quimioterapia no paciente. Nesse trabalho, Pinho e colaboradores (Pinho et al., 2002), consideram $\Delta$ constante, o que representa uma infusão contínua de quimioterapia. Alguns trabalhos já simularam em seus modelos para câncer a quimioterapia em doses (Ahn and Park, 2011; Engelhart et al., 2011; Villasana et al., 2010) onde a taxa de infusão do AQ não é nula apenas no momento da aplicação.

Neste trabalho unimos as ideias de Banerjee e Sarkar ((Banerjee and Sarkar, 2008)) e Pinho e colaboradores ((Pinho et al., 2002)) para formular um modelo mais completo que considera a interação do câncer com o sistema imunológico (Banerjee and Sarkar, 2008) e também a quimioterapia (Pinho et al., 2002). Outros modelos da literatura também consideram no modelo para câncer os linfócitos e a quimioterapia (Pillis and Randunskaya, 1999; Villasana and Radunskaya, 2003; Liu et al., 2007), porém esses modelos não consideram os linfócitos em duas formas (caça e repouso). Os linfócitos em repouso são fundamentais na ativação dos linfócitos em caça e como a quimioterapia atua como predador tanto no câncer como nos linfócitos é muito importante considerar num modelo pra câncer com quimioterapia os linfócitos em repouso. Analisou-se numericamente o comportamento dinâmico do crescimento do câncer variando alguns parâmetros do sistema relacionados ao tempo de atraso e a quimioterapia.

O presente trabalho está segmentado da seguinte forma: na seção 2 descreve-se o modelo, na seção 3 mostra-se análises numéricas do modelo para diferentes formas de quimioterapia e na seção 4 tem-se as conclusões.

\section{Modelo}

Modificamos o modelo matemático proposto por Banerjee and Sarkar (Banerjee and Sarkar, 2008) incluindo termos que representam um agente quimioterápico. Este agente é inserido como um predador tanto no câncer como nos linfócitos. A Figura 1 mostra um esquema das interações. Há um tempo de atraso na ativação e conversão dos linfócitos em repouso para linfócitos em caça. Representou-se por $C$ o número de células cancerígenas, $H$ o número de linfócitos em caça, $R$ o números de linfócitos em repouso e $Z$ a quantidade de agente quimioterápico (AQ). O modelo é dado pelas seguintes equações

$$
\begin{aligned}
& \frac{d C(t)}{d t}=q_{1} C(t)\left(1-\frac{C(t)}{K_{1}}\right) \\
& -\alpha_{1} C(t) H(t)-\left(\frac{p_{1} C(t)}{a_{1}+C(t)}\right) Z(t) \\
& \frac{d H(t)}{d t}=\beta_{1} H(t) R(t-\tau)-d_{1} H(t) \\
& -\alpha_{2} C(t) H(t)-\left(\frac{p_{2} H(t)}{a_{2}+H(t)}\right) Z(t) \\
& \frac{d R(t)}{d t}=q_{2} R(t)\left(1-\frac{R(t)}{K_{2}}\right) \\
& -\beta_{1} H(t) R(t-\tau)-\left(\frac{p_{3} R(t)}{a_{3}+R(t)}\right) Z(t) \\
& \frac{d Z(t)}{d t}=\Delta-\left(\xi+\frac{g_{1} C(t)}{a_{1}+C(t)}\right. \\
& \left.+\frac{g_{2} H(t)}{a_{2}+H(t)}+\frac{g_{3} R(t)}{a_{3}+R(t)}\right) Z(t),
\end{aligned}
$$

onde $C(0)=C_{0}>0, H(0)=H_{0} \geq 0, R(0)=$ $R_{0}>0, Z(0)=Z_{0} \geq 0$. O termo $-d_{1} H(t)$ representa a morte natural dos linfócitos em caça, o termo $-\alpha_{1} C(t) H(t)$ representa o decréscimo de células tumorais devido ao encontro com os linfócitos em caça e $-\alpha_{2} C(t) H(t)$ o decréscimo dos linfócitos em caça devido ao encontro com as células do tumor. Há um atraso na conversão dos linfócitos em repouso para os linfócitos em caça, que explica o termo $-\beta_{1} H(t) R(t-\tau)$ na terceira equação. Este atraso na transformação induz um atraso no crescimento dos linfócitos em caça, o que 
justifica o termo $\beta_{1} H(t) R(t-\tau)$ na segunda equação. O último termo das três primeiras equações representam a eliminação das células pelo AQ. Os parâmetros utilizados no modelo foram:

- $q_{1}, q_{2}$ : taxas de proliferação das células tumorais e dos linfócitos em repouso, respectivamente;

- $K_{1}, K_{2}$ : capacidades bióticas máximas das células tumorais e dos linfócitos em repouso, respectivamente;

- $\alpha_{1}, \alpha_{2}$ : coeficientes de competição entre $C$ e $H$;

- $p_{1}, p_{2}, p_{3}$ : coeficientes de predação de $Z$ em $C, H$ e $R$;

- $a_{1}, a_{2}, a_{3}$ : determinam as velocidades com que $C, H$ e $R$, na ausência de competição e predação, atinge a capacidade de suporte;

- $\Delta$ : taxa de infusão do AQ;

- $\xi$ : taxa de eliminação do AQ;

- $g_{1}, g_{2}, g_{3}$ : taxas de combinação do AQ com as células;

- $\tau$ : atrazo de tempo na conversão dos linfócitos em repouso para linfócitos em caça.

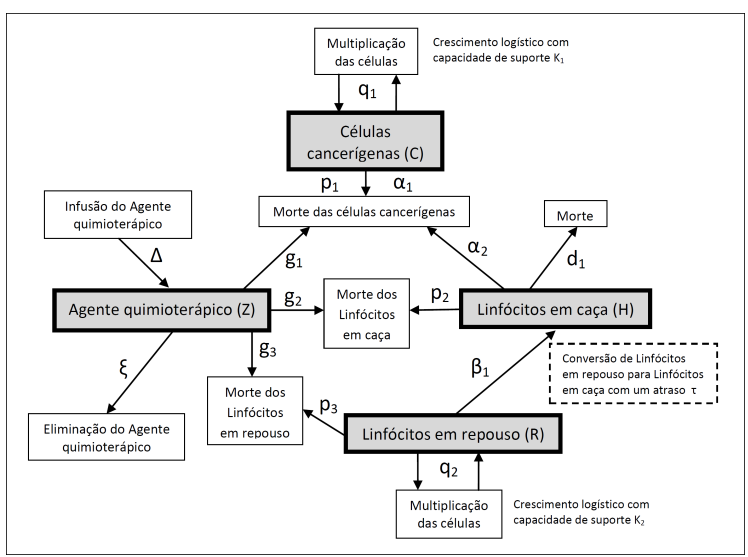

Figura 1: Representação esquemática das interações do modelo 1.

Os parâmetros do modelo 1 foram obtidos por meio de experimentos com camundongos e por considerações matemáticas. Para deixar o modelo mais geral e estudar apenas o comportamento das soluções fazemos uma adimensionalização. Com isso, não é necessário preocupar-se com as unidades de medida de $C, H, R$ e $Z$. Adimensionalizouse o sistema 1 definindo-se: $\bar{t}=t / \operatorname{dia}, \bar{C}=C / K_{T}$, $\bar{H}=H / K_{T}, \bar{R}=R / K_{T}, \bar{Z}=Z / \Delta_{M} \xi^{-1}$, onde $K_{T}=K_{1}+K_{2}$ é a capacidade de suporte total e $\Delta_{M}$ é igual a $1 \mathrm{mg} \mathrm{m}^{-2} \mathrm{dia}^{-1}$. Substituindo essas variáveis admensionais no sistema 1 , e renomeando as variáveis $\bar{t}, \bar{C}, \bar{H}, \bar{R}, \bar{Z}$ como $t, C, H, R$,
$Z$, respectivamente, e os parâmetros $\bar{q}_{1}, \bar{K}_{1}, \bar{\alpha}_{1}$, $\bar{p}_{1}, \bar{g}_{1}, \bar{a}_{1}, \bar{\beta}_{1}, \bar{d}_{1}, \bar{\alpha}_{2}, \bar{p}_{2}, \bar{g}_{2}, \bar{a}_{2}, \bar{q}_{2}, \bar{K}_{2}, \bar{p}_{3}, \bar{g}_{3}, \bar{a}_{3}$, $\bar{\Delta}, \bar{\xi}$ como $q_{1}, K_{1}, \alpha_{1}, p_{1}, g_{1}, a_{1}, \beta_{1}, d_{1}, \alpha_{2}, p_{2}$, $g_{2}, a_{2}, q_{2}, K_{2}, p_{3}, g_{3}, a_{3}, \Delta$, $\xi$, respectivamente, obtêm-se as mesmas equações para $C, H$ e $R$. A equação para $Z$ exibe uma alteração,

$$
\left.\begin{array}{l}
\frac{d Z(t)}{d t}=\Delta \xi-\left(\xi+\frac{g_{1} C(t)}{a_{1}+C(t)}+\frac{g_{2} H(t)}{a_{2}+H(t)}\right. \\
\left.+\frac{g_{3} R(t)}{a_{3}+R(t)}\right) Z(t),
\end{array}\right\}
$$

onde consideramos

$$
\begin{array}{ll}
\bar{q}_{1}=q_{1} \text { dia } & \bar{\alpha}_{1}=\alpha_{1} K_{T} \text { dia } \\
\bar{K}_{1}=\frac{K_{1}}{K_{T}} & \bar{p}_{1}=\frac{p_{1} \Delta_{M} \text { dia }}{K_{T} \xi} \\
\bar{a}_{1}=\frac{a_{1}}{K_{T}} & \bar{\beta}_{1}=\beta_{1} K_{T} \text { dia } \\
\bar{d}_{1}=d_{1} \text { dia } & \bar{\alpha}_{2}=\alpha_{2} K_{T} \text { dia } \\
\bar{g}_{1}=g_{1} \text { dia } & \bar{g}_{2}=g_{2} \text { dia } \\
\bar{g}_{3}=g_{3} \text { dia } & \bar{p}_{2}=\frac{p_{2} \Delta_{M} \text { dia }}{K_{T} \xi} \\
\bar{a}_{2}=\frac{a_{2}}{K_{T}} & \bar{K}_{2}=\frac{K_{2}}{K_{T}} \\
\bar{p}_{3}=\frac{p_{3} \Delta_{M} \text { dia }}{K_{T} \xi} & \bar{a}_{3}=\frac{a_{3}}{K_{T}} \\
\bar{\Delta}=\frac{\Delta}{\Delta_{M}} & \bar{q}_{2}=q_{2} \text { dia } \\
\bar{\xi}=\xi \text { dia. } &
\end{array}
$$

Usando as relações apresentadas em (3) obtêm-se os valores para os parâmetros adimensionalizados apresentados na tabela 1. Considerouse que a predação da quimioterapia no câncer e nos linfócitos tem a mesma intensidade (Villasana and Radunskaya, 2003). Os parâmetros $\Delta$ (taxa de infusão do AQ) e $p_{i}$ (coeficiente de predação do $\mathrm{AQ}$ ) dependem da quantidade e do tipo de quimioterapia que é aplicada ao paciente. Estudou-se o comportamento do modelo para uma determinada faixa desses parâmetros $\left(\Delta\right.$ de 0 até $10^{4}$ e $p_{i}$ de $10^{-8}$ até 1 ). O parâmetro $g_{i}$ (taxa de combinação do $\mathrm{AQ}$ com as células) é proporcional à $p_{i}$ (Pinho et al., 2002).

Tabela 1: Valores dos parâmetros adimensionalizados

\begin{tabular}{c|c|c|c} 
Parâmetro & Valor & Parâmetro & Valor \\
\hline$q_{1}$ & 0,18 & $K_{1}$ & $1 / 3$ \\
$\alpha_{1}$ & 1,6515 & $\alpha_{2}$ & $5,13 \times 10^{-3}$ \\
$d_{1}$ & 0,0412 & $q_{2}$ & 0,0245 \\
$\tau$ & 45,6 & $K_{2}$ & $2 / 3$ \\
$\beta_{1}$ & $9,3 \times 10^{-2}$ & $p_{1}$ & $1,0 \times 10^{-3}$ \\
$p_{2}$ & $1,0 \times 10^{-3}$ & $p_{3}$ & $1,0 \times 10^{-3}$ \\
$a_{1}$ & $1,0 \times 10^{-4}$ & $a_{2}$ & $1,0 \times 10^{-4}$ \\
$a_{3}$ & $1,0 \times 10^{-4}$ & $g_{1}$ & 0,1 \\
$g_{2}$ & 0,1 & $g_{3}$ & 0,1 \\
$\Delta$ & $0-10^{4}$ & $\xi$ & 0,2
\end{tabular}

\section{Análises numéricas}

Estudou-se numericamente o modelo para verificar o comportamento das soluções e analisar a quimioterapia necessária para suprimir o câncer sem acabar com os linfócitos. Considerou-se duas formas de quimioterapia: constante e em doses. 

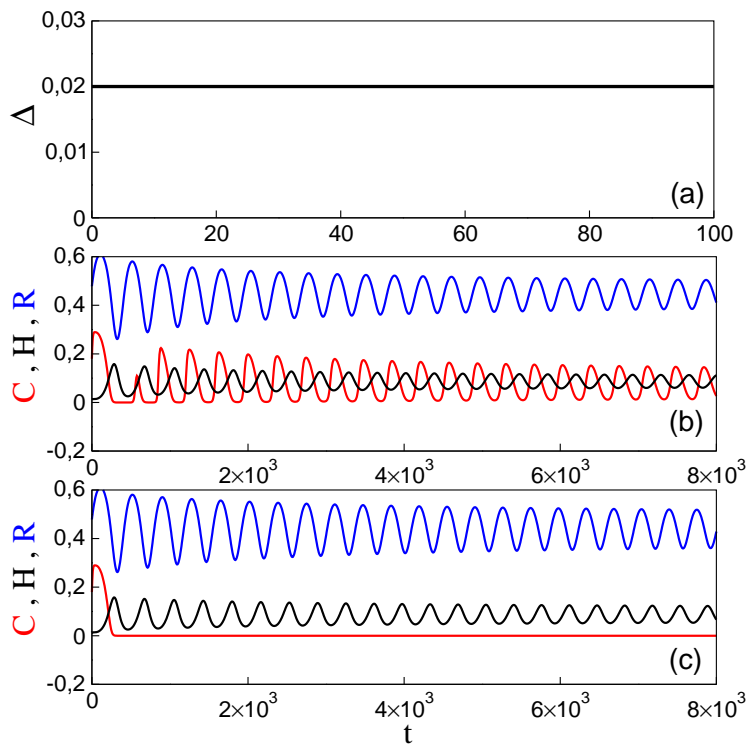

Figura 2: Evolução temporal das células. (a) Infusão contínua de quimioterapia, (b) $\Delta=0,02 \mathrm{e}$ (c) $\Delta=0,022$. A linha vermelha representa as células cancerígenas, a linha preta os linfócitos de caça e a linha azul os linfócitos em repouso.

Utilizou-se as condições iniciais adimensionalizadas: $C_{0}=0,18, H_{0}=0,0136, R_{0}=0.4786 \mathrm{e}$ $Z_{0}=0$ (Banerjee and Sarkar, 2008). Uma célula de câncer equivale a $6,6 \times 10^{-8}$ no modelo adimensionalizado. Considerou-se como supressão do câncer, o caso onde haja menos do que uma célula.

Analisou-se primeiramente o caso com a taxa de infusão de quimioterapia $(\Delta)$ como sendo uma constante. Na figura 2 tem-se a evolução temporal das células. Na figura 2(a) mostra-se o comportamento da taxa de infusão de quimioterapia. Quando $\Delta=0,02$ não há supressão do câncer e o sistema exibe um estado oscilatório estável (figura 2(b)). Aumentando-se esse valor para $\Delta=0,022$ o sistema apresenta um estado onde há eliminação do câncer sem grandes perdas nas quantidades de linfócitos (figura 2(b)).

Verificou-se que a supressão ou não do câncer depende do coeficiente de predação do AQ $\left(p_{1}\right)$ e da taxa de infusão do AQ $(\Delta)$. A figura 3 mostra essa dependência, onde pode-se verificar a existência de três regiões. Na região branca, não há supressão do câncer, os valores de $p_{1}$ e $\Delta$ não são suficientes para que ocorra a eliminação de todas as células cancerígenas. Na região cinza tem-se a situação em que a quimioterapia elimina todos os linfócitos, o que não é interessante clinicamente. Focaremos nossos estudos na região preta, onde há supressão do câncer sem eliminação de todos os linfócitos. Para analisar o comportamento do nosso modelo em função de $\Delta$, fixaremos o valor de $p_{1}=10^{-3}$.

Além de eliminar o câncer, deseja-se que isso ocorra em um menor tempo possível. Observou-se

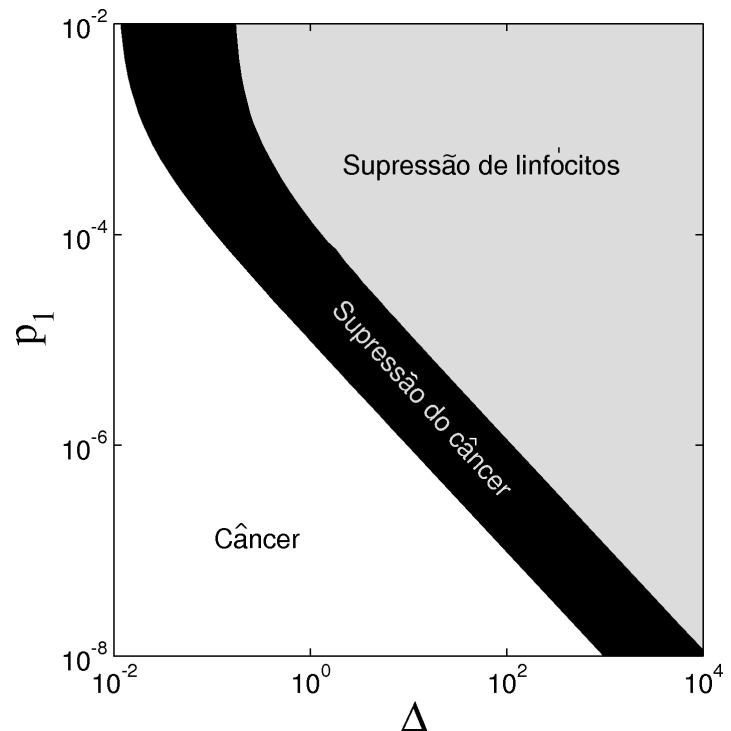

Figura 3: Coeficiente de predação do AQ nas células cancerígenas em função da taxa de infusão do AQ. Região em preto ocorre a supressão do câncer, região em branco não ocorre a supressão do câncer e região em cinza ocorre a supressão de linfócitos. Os parâmetros utilizados estão na tabela 1 .

que temos um valor mínimo para a taxa de infusão de AQ $(\Delta)$ em que o câncer é suprimido. Analisouse o quanto $\Delta$, acima deste mínimo, pode diminuir o tempo de supressão. Quando $\Delta$ é igual a $2,2 \times 10^{-2}$ a supressão ocorre em $t=636,6$. O tempo de supressão do câncer $(T)$ diminui até $\Delta=0,07$, onde $T$ assume um valor mínimo $T_{\text {min }}=338$ (figura 3 ). Para valores de $\Delta$ superiores a 0,07 até 0,266 o aumento de $\Delta$ causa em $T$ um acréscimo. Esse aumento no tempo de supressão do câncer com uma maior taxa de infusão de AQ pode ser efeito da grande destruição dos linfócitos, que é causada para grandes valores de $\Delta$. Os linfócitos agem como predadores do câncer e uma diminuição no seu número implica em uma menor mortalidade das células cancerígenas pelo sistema imunológico. Quando $\Delta$ é aproximadamente 0,266 há uma supressão dos linfócitos, deixando a quimioterapia como o único predador do câncer, o que faz o tempo de supressão aumentar.

Analisou-se também o caso onde consideramos a taxa de infusão de quimioterapia $(\Delta)$ em doses periódicas com período $P$ e amplitude $\Delta_{1}$, onde a taxa de infusão do AQ é diferente de zero apenas no dia da aplicação da quimioterapia (figura 3(a)). Aplicando-se a quimioterapia a cada 10 unidades de tempo $(P=10)$ verificamos que quando $\Delta_{1}=0,20$ não há supressão do câncer (figura $3(\mathrm{~b}))$. É necessário que $\Delta_{1}$ seja aproximadamente 0,22 para eliminar as células cancerígenas (figura $3(\mathrm{c})$ ).

Notou-se um comportamento linear da quan- 


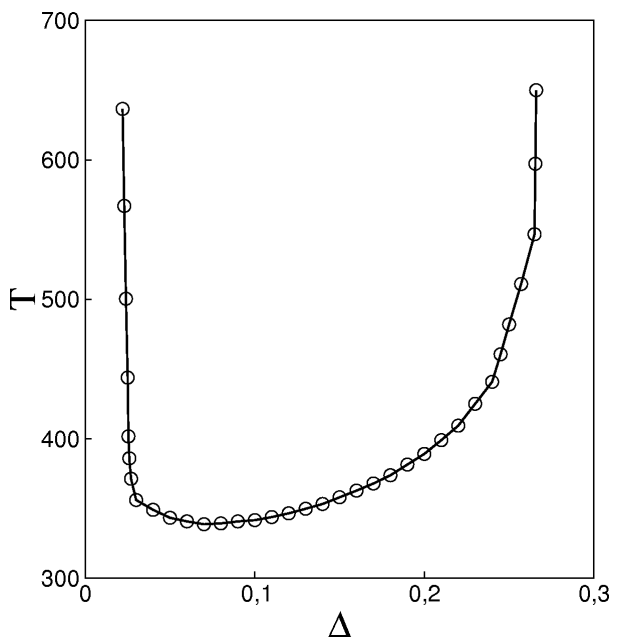

Figura 4: Tempo de supressão do câncer em função da taxa de infusão do AQ. Os parâmetros utilizados estão na tabela 1.

tidade mínima necessária de $\Delta_{1}$ para suprimir o câncer em função do período entre as doses $P$ (figura 3 ). Dobrando-se o intervalo de tempo entre as doses de quimioterapia precisou-se também dobrar o valor de $\Delta_{1}$ para se obter o mesmo efeito.

\section{Conclusões}

Nesse trabalho, foram estudadas as interações entre células cancerígenas e o sistema imunológico quando submetidos a quimioterapia por meio de um sistema de equações diferenciais não-lineares com atraso. O modelo proposto é simples e geral. Para deixar o modelo mais abrangente foi feita uma adimensionalização, que possibilitou estudálo sem se preocupar com as unidades de medida das grandezas.

Verificou-se que para uma quantidade pequena da taxa de infusão do agente quimioterápico $(\Delta)$ a quantidade de células cancerígenas, de linfócitos em caça e de linfócitos em repouso não são afetadas significativamente. A partir de um determinado valor de $\Delta$ há uma eliminação de todas as células. Observou-se que para uma determinda faixa de valores da quimioterapia o modelo apresenta um comportamento que é mais interresante clinicamente, que é a supressão do câncer sem a destruição do sistema imunológico. Foi estudada essa supressão do câncer para dois tipos de taxa de infusão do agente quimioterápico $(\Delta)$ : constante e em doses.

Para $\Delta$ constante delimitou-se a região onde há supressão do câncer em função dos parâmetros da quimioterapia $p_{1}$ (coeficiente de predação do agente quimioterápico $(\mathrm{AQ}))$ e $\Delta$. Isto possibilitou fixar o valor de $p_{1}$. Obteve-se valores mínimos da quimioterapia que suprimem o câncer e que para essas quantidades o tempo de supressão é relativamente grande. Observou-se que há um

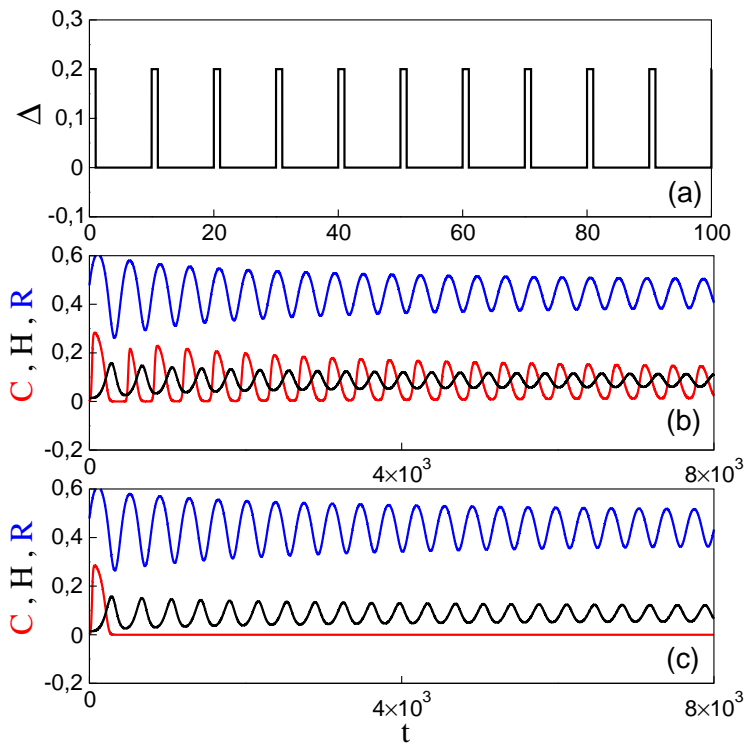

Figura 5: (a) $\Delta \times t-\mathrm{A}$ taxa de infusão do AQ é diferente de zero apenas no dia da aplicação da quimioterapia. (b)Evolução temporal das células com aplicação de quimioterapia em 10/10 dias com $\Delta_{1}=0,2$. (c)Evolução temporal das células com aplicação de quimioterapia em 10/10 dias com $\Delta_{1}=0,22$.

valor de quimioterapia em que o tempo supressão do câncer é mínimo e que para valores acima deste o tempo de supressão aumenta.

Verificou-se que, para taxa de infusão do agente quimioterápico em doses constantes, há uma dose mínima de quimioterapia que suprime o câncer e a supressão depende do período entre as doses $(P)$. Quanto maior for a dose de quimioterapia $\left(\Delta_{1}\right)$ maior pode ser o tempo entre as doses necessário para suprimir o câncer. Notou-se que esse comportamento de $\Delta_{1}$ em função de $P$ para a supressão do câncer é linear. Se dobrarmos o intervalo de tempo entre as doses de quimioterapia precisamos também dobrar o valor de $\Delta_{1}$ para obter o mesmo efeito.

\section{Agradecimentos}

A realização do trabalho foi possível devido ao auxílio financeiro das seguintes agências: CNPq, CAPES e Fundação Araucária.

\section{Referências}

Ahn, I. and Park, J. (2011). Drug scheduling of cancer chemotherapy based on natural actor-critic approach, BioSystems 106: 121129. DOI: 10.1016/j.biosystems.2011.07.005

Banerjee, S. and Sarkar, R. R. (2008). Delayinduced model for tumor-immune interaction and control of malignant tumor growth, $B i$ oSystems 91: 268-288. DOI: 10.1016/j.biosystems.2007.10.002 


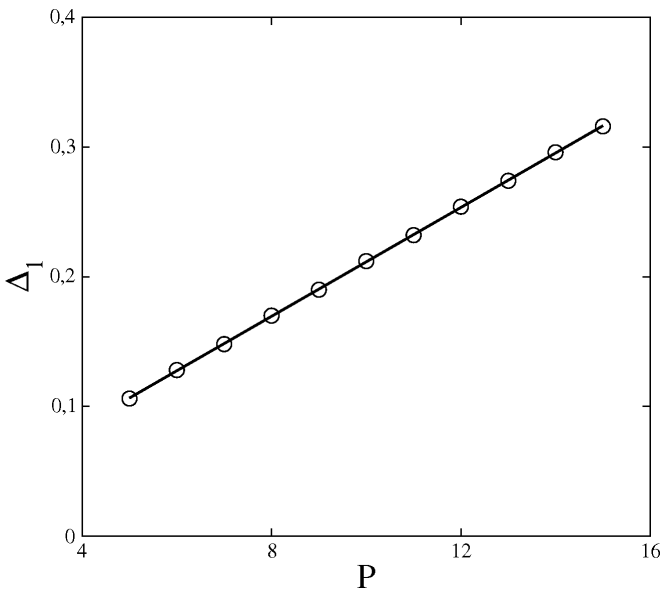

Figura 6: Quantidade mínima necessária de $\Delta_{1}$ para suprimir o câncer em função do período entre as doses $P$. A linha preta representa uma regressão linear dos pontos.

Dorr, R. T. and VonHoff, D. D. (1994). Cancer Chemotherapy Handbook, Appleton \& Lange, Connecticut.

Engelhart, M., Lebiedz, D. and Sager, S. (2011). Optimal control for selected cancer chemotherapy ode models: A view on the potential of optimal schedules and choice of objective function, Math. Biosc. 229: 123-134. DOI: $10.1016 /$ j.mbs.2010.11.007

Iarosz, K. C., Martins, C. C., Batista, A. M., Viana, R. L., Lopes, S. R., Caldas, I. L. and Penna, T. J. P. (2011). On a cellular automaton with time delay for modelling cancer tumors, J. Phys.: Conf. Ser. 285(012015).

INCA (2011). Tipos de câncer, Disponível em: <www.inca.gov.br>. Acesso em: 03 de março de 2011.

Liu, W. and Freedman, H. I. (2005). A mathematical model of vascular tumor treatment by chemotherapy, Mathematical and Computer Modelling 42: 1089-1112. DOI: 10.1016/j.mcm.2004.09.008

Liu, W., Hillen, T. and Freedman, H. I. (2007). A mathematical model for m-phase specific chemotherapy including the g0-phase and immunoresponse, Mathematical Biosciences and Engineering 4: 239-259. DOI: 10.3934/mbe.2007.4.239

Otto, S. E. (2002). Oncologia, Reichmann and Affonso, Rio de Janeiro.

Pillis, L. G., Gu, W. and Randunskaya, A. (2006). Mixed immunotherapy and chemothe-rapy of tumors: modeling, applications and biological interpretations, J. of Theor. Biol. 238: 841-862. DOI: 10.1016/j.jtbi.2005.06.037

Pillis, L. G. and Randunskaya, A. (1999). A mathematical tumor model with immune re- sistance and drug therapy: an optimal control approach, Harvey Mudd College and Pomona College.

Pinho, S. T. R., Freedman, H. I. and Nani, F. (2002). A chemotherapy model for the treatment of cancer with metastasis, Mathemati-cal and Computer Modelling 36: 773-803. DOI: 10.1016/S0895-7177(02)00227-3

Sarkar, R. R. and Banerjee, S. (2005). Cancer self remision and tumor stability - a stochastic approach, Math. Biosci. 196: 65-81. DOI: 10.1016/j.mbs.2005.04.001

Villasana, M., Ochoa, G. and Aguilar, S. (2010). Modeling and optimization of combined cytostatic and cytotoxic cancer chemotherapy, Artificial Intelligence in Medicine 50: 163173. DOI: $10.1016 /$ j.artmed.2010.05.009

Villasana, M. and Radunskaya, A. (2003). A delay differential equation model for tumor growth, J. Math. Biol. 47: 270-294. DOI:

Wodarz, D., Klenerman, P. and Nowak, M. (1998). Dynamics of cytotoxic t-lymphocyte exhaustion, Proc. R. Soc. Lond. B 265: 191203. DOI: $10.1098 / \mathrm{rspb} .1998 .0282$ 